\title{
Bridging the Gap: Exploring Interactions Between Digital Human Models and Cognitive Models
}

\author{
Robert G. Feyen \\ Department of Mechanical and Industrial Engineering \\ University of Minnesota - Duluth \\ rfeyen@d.umn.edu
}

\begin{abstract}
For years now, most researchers modeling physical and cognitive behavior have focused on one area or the other, dividing human performance into "neck up" and "neck down." But the current state of the art in both areas has advanced to the point that researchers should begin considering how the two areas interact to produce behaviors. In light of this, some common terms are defined so researchers working in different disciplines and application areas can understand each other better. Second, a crude "roadmap" is presented to suggest areas of interaction where researchers developing digital human form and other physical performance models might be able to collaborate with researchers developing cognitive models of human performance in order to advance the "state-of-the-art" in replicating and predicting human performance.
\end{abstract}

Keywords: Human performance, cognitive models, digital human models.

"The modeling of cognition and action by individuals and groups is possibly the most difficult task humans have yet undertaken. Developments in this area are still in their infancy. Yet, important progress has been and will continue to be made." [1]

\section{Introduction}

Indeed, considerable progress is being made in our ability to model humans and their behavior. With the improvements in computing technology over the past three decades, we have been fortunate to have researchers able to take advantage of the increases in processing speeds, graphics capabilities, and computational power in order to generate digital human form models with increasing levels of realism. The same improvements have allowed other researchers to specify computational models that predict a human's physical performance and cognitive function with surprising levels of accuracy.

However, the claim made above that work in this area is still in its infancy may be more apt than ever. Those familiar with child development might observe that our digital human form models have less cognitive abilities than an infant and, in large part, are still developing the neuromuscular patterns for coordinating and executing limb and other body motions. They might also observe that our cognitive models still have difficulty perceiving much of the environment around them and figuring out 
what to do in response without us helping them out by telling them what's going on around them and what they should do. Finally, they would likely observe that these models typically are developed and used separately from one another.

They might then argue that, since physical and cognitive development in an infant are intertwined, perhaps it would make sense to stop dividing human performance into "neck up" and "neck down" (a distinction made by many in human factors and ergonomics between cognitive and physical performance) and instead begin considering how the two interact to produce behaviors. However, as many things in research and life, this is far easier said than done. But the argument has strong merit; thus, the two main goals of this opinion paper are to (1) define some common terms so researchers working in different disciplines and application areas can understand each other better and (2) present a crude "roadmap" that suggests areas of interaction where researchers developing digital human form and other physical performance models and researchers developing cognitive models of human performance might be able to collaborate in order to advance the "state-of-the-art" in replicating and predicting human performance.

\section{Defining Common Terminology}

For over a century now, researchers have been working to understand the nature of the various interactions between man and machine while engineers and designers have been attempting to apply this understanding to the design of products and systems. But to do this effectively, the engineers and designers must be able to readily access and utilize this information in the earliest stages of the design process. Designers already have access to tools that can predict hardware and software performance; but to consider human-machine interaction in the conceptual stages of a design, they need tools that can predict how the human will perform using task-based metrics associated with the speed, actions, timing, and errors that may arise while undertaking a task.

Such tools often are called "human performance models" or HPMs. HPMs can be found throughout a diverse range of disciplines and all attempt to represent or describe some aspect of human performance. Disciplines include psychology, cognitive science, engineering, artificial intelligence, computer science, biomechanics, physiology, and medicine. HPMs have been used in the design of complex systems ranging from industrial processes and nuclear power plants to surface and air transportation systems; they have also been used extensively in the field of human-computer interaction. Types of human performance modeled by these approaches include perceptual functions such as signal detection, visual scanning, object recognition, and motion estimation as well as models of various cognitive functions including problem solving, memory recall, training, skill acquisition, and errors. Other approaches consider a person's anthropometric and biomechanical characteristics while yet other approaches provide insight into the workload - both mental and physical - experienced by a person. Some approaches distinguish between individual operators and group operations (e.g., multi-operator systems such as found in passenger jet cockpits) while others distinguish between performance on a single task and performance in situations where several tasks must be completed at the same time. This diverse range of applications and disciplines makes discussing tools for 
predicting human performance difficult since the same terminology often has different meanings, depending on who is using the tool.

To avoid confusion, the terms "approach", "framework" (or, "architecture"), and "model" should be defined separately since they are often used interchangeably in the HPM literature and commonly confused.

- An approach encompasses the methodology used to specify the task, environment (including equipment), and human in a consistent manner and the means by which this information is incorporated into the modeling efforts to generate measures of performance.

- The framework is a general structure that contains elements and parameters common to any scenario to be modeled (e.g., information processors and their default service times). The task, environment, and human information as specified by the approach indicate which elements of the framework are required and which parameters must be altered when modeling a specific system scenario.

- The resulting structural elements and the set of parameters representing a specific system scenario are called the model.

Another area of confusion pertains to the output of human performance approaches: these approaches yield engineering models, not psychological models. In the psychological literature, a "human performance model" is narrowly focused, with the term often used synonymously with a theory of performance. Typically, a human performance model in the psychological literature is independent of the system or task contexts in order to be applicable in a wide range of settings. A few of these models tend to be analytic and limited in scope with an exact solution for a given set of parameters (e.g., Fitt's law) while the majority of others are qualitative models (e.g., principles of stimulus-response compatibility). However, their primary purpose is to evaluate and develop the theory underlying the model. When a model does not agree with data obtained or behaviors observed from the real world, the model and theory are either discarded or reworked until agreement is reached. This differs from the engineering approaches in which, regardless of the characteristics of a particular model, a model has value as an engineering or design tool as long as the model can aid decision making by yielding simulated performance data that approximates real performance data within acceptable limits.

Yet another complicating issue is defining what one means by "model". For example, researchers in one discipline may conceive of models that differ dramatically from what researchers in another discipline might call a model. However, Baron et al [2] and Elkind et al [3] have proposed useful classifications to help bridge that gap. In particular, they identified eight (8) pairs of characteristics that can be used to define the purpose of a given model:

1. A predictive model is capable of accurate performance predictions without requiring extensive validation data. On the other hand, the parameters of a descriptive model must be adjusted in order to fit the model to existing performance data. Obviously, a predictive model is more useful to a decisionmaker and, if accurate, also classifies as a descriptive model.

2. Primarily focused on system performance, a top-down model starts with a set of system goals, which are then decomposed into progressively smaller sub-goals and 
processes until a level is reached at which the resulting processes are deemed as "primitives" (which cannot be broken down any further). A bottom-up model starts with a predefined set of primitives and links various primitives together to form an overall system. This approach concentrates primarily on the process that dictates the system's performance, although the performance itself is also considered.

3. Simulation models imitate human performance and, by manipulating the inputs and outputs, allow interactions between the human, the system, and the environment to be simulated and analyzed. Analytic models represent human performance mathematically, providing concise descriptions that in some cases may be referred to as "laws" of human behavior.

4. A process model attempts to predict the human output by representing the specific human processes used to accomplish the task. A performance (or, output) model attempts to predict performance without any consideration of the human processes involved and places no requirements on the structure or validity of the inner workings of the model. In many situations, output models are sufficient for the purposes of analysis; but, since process models are applicable to a wider range of tasks and conditions, they are the more powerful of the two.

5. A descriptive model describes the current human behavior, performance, or process while a prescriptive model (also known as normative or rational action) suggests the human behavior or process that will lead to optimal performance.

6. A model may have either depth or breadth, depending on the amount of detail desired and the level at which behavior is being modeled.

7. Elkind et al [3] define a model focused on a single task as limited whereas comprehensive refers to a model capable of addressing a variety of different tasks. However, this definition can be expanded to include the range of human performance issues addressed by an HPMA. By necessity, early attempts at formulating HPMAs addressed only limited aspects of human performance (e.g., visual scanning or strength capabilities) since modeling multiple aspects was too complex and time-consuming to evaluate. But continued research combined with rapid advances in computer technology has rekindled efforts to integrate numerous aspects of human behavior into a single, coherent modeling framework. For example, recent comprehensive approaches such as SOAR [4], ACT-R [5], MIDAS [6], EPIC [7] and the QN-MHP [8] are capable of predicting certain aspects of performance accurately across an increasingly wider range of tasks and human behaviors.

8. While qualitative models based on verbal theories and empirical data can be useful in analyzing human performance, they are not especially useful for performance prediction. Some authors refer to certain quantitative models as "formal models", defined as those arising from one of two distinctly different types of approach: those based on mathematical theories and those based on computational techniques. ${ }^{1}$

${ }^{1}$ In March 2001, the Human Factors journal published a call for participation regarding a special issue to be titled "Quantitative Formal Models of Human Performance." In the call, the co-editors (Wayne Gray and Michael Byrne) state “...'Formal models' are construed to include both computational and mathematical models..." 
Even within these latter two approaches to quantitative models, different conceptual frameworks are used to elicit measures of human performance. For example, linear statistical and control theoretic frameworks can be identified separately within the mathematical category while information processing, task networking and knowledge-based frameworks fall under the computational umbrella $[9,10]$. This delineation is not absolute as recent advances in many frameworks borrow features or techniques from various other frameworks. This trend towards integration undoubtedly will continue as no single approach is likely to provide a comprehensive general model capable of describing or predicting human behavior in various task settings (see Gluck and Pew [11] for an excellent review of the state-ofthe-art in integrated approaches).

\section{Interactions Between Physical and Cognitive Models}

For this same reason, physical and cognitive models of human performance also need to be integrated. Approaches such as HOS [12] and MIDAS [13] as well as work by researchers at Sandia National Laboratories [14] represent initial efforts to integrate physical and cognitive models into a single approach by including independent "modules" (micromodels containing algorithms based on human experimental literature) that can be used as necessary to predict an aspect of performance. One criticism is that performance predicted by these "plug and play" modules actually may not be independent; that is, interactions between different aspects of performance may produce behaviors and performance that the modular approach would have difficulty predicting accurately.

But is this criticism valid? The answer is - no one seems to know. Published research on the interactions between various models is scarce, if not nonexistent. What interactions need to be studied? Space does not permit a comprehensive discussion of this, but three major types of interaction warrant further attention physical, physiological, and emotional. But before discussing these, three external influences impacting these interactions of cognitive and physical performance should be noted: the activities of the person, the equipment available to the person for carrying out these activities, and the environment in which the person acts.

\subsection{External Influences Impacting Interactions}

The first external influence is the person's activities. This encompasses more than the elements of a specific task that a person may be attempting to complete. Models of task performance often fail to capture the full range of activities undertaken by a human, primarily because these tasks occur in a broader context which may include elements unrelated to the task at hand. The source of these "other" activities may be rooted in the person (e.g., physiological needs requiring some response or prospective memory leading to performance of another task), the situational context (e.g., task deadlines or other externally defined performance requirements, safety implications, local events), or the environment (e.g., workplace layout, climate, ecology). Further, these activities impact the person's physiological system, having effects that may 
accumulate over time (both short term and long term) and trigger a physiological response that must be addressed by an activity that interrupts the current task.

The second external influence is the equipment available for use by the person to carry out these or other activities. Once again, not all of the available equipment may be used by a person in a given location or time frame to complete the task at hand, but may simply be present. However, such equipment has potential to produce distractions or interruptions leading to activities unrelated to the immediate task at hand. For example, telephones, e-mail and instant messaging alerts, instrument displays, and alarm monitors can produce responses that detract from the performance of the task at hand. Secondly, many activities can be completed satisfactorily using different types of available equipment, requiring the person to choose between these options and the resulting strategies in order to complete the chosen activity. For example, imagine the various ways in which people can write reminders to themselves. At any given time, the method selected often depends on the type of writing surface available (sticky notes, scrap paper, one's hand), type of writing utensils (pens, pencils, markers), computer or phone access (send oneself an e-mail or voicemail), available surfaces for attaching notes (e.g., bulletin boards, whiteboards, walls), etc.

The person's environment acts as the third external influence. The workplace itself may hinder or facilitate activities, depending on housekeeping practices as well as the organization and location of equipment and objects that must be seen, heard, or manipulated. Activities taking place in warm, dry conditions may require additional steps or entirely different methods if carried out in cold, wet conditions. As with activities, physiological responses to environmental conditions often detract from task performance and may lead to interruptions as the person carries out an activity unrelated to the task at hand in order to address a basic physiological need. Other environmental conditions such as noise, poor surface conditions, vibration, rain and sunlight often produce responses extraneous to those needed to complete a given task or lead to errors in the performance of the task.

These three influences (activity, equipment, and environment) should not be surprising to anyone working in the area of either digital human (physical) or cognitive modeling. But what becomes apparent in this discussion is the strong bidirectional relationship between the physical and cognitive aspects of human performance. At all times, a person's actions and physical presence in the environment and perceived proximity to nearby objects along with the person's internal physiological responses generates information used by the cognitive system to determine what to do next. Taking this diverse information into account with the situational context, the cognitive system attempts to select a strategy likely to be feasible, successful, and appropriate for that moment in time. For example, importance of an activity, the available time, the time required to complete an activity, and the individual's appraisal of their ability to complete an activity successfully (i.e., self-efficacy) are significant factors impacting which activity or set of activities a person chooses to do at any given time [15, 16, 17, 18]. However, once a strategy is selected, the cognitive system must also dictate how the physical system will respond while accounting for the person's functional limitations, equipment capabilities, and environmental constraints. Once initiated, this response impacts the status of all activities currently underway, alters the environment, and affects the 
body's physiology and its position relative to any available equipment. And the cycle repeats. Notably, this iterative balancing act implies that not all activities carried out by an individual will be rational, at least not in the narrow context of achieving a single task-oriented goal as assumed in many cognitive modeling approaches. Thus, to improve our ability to model human performance, we need a better understanding of how these various sources of information influence the choice of activities, strategies and responses - and vice versa.

\subsection{Interactions Between Models}

\subsubsection{Physical Interactions}

Performance monitoring. From the digital human modeling standpoint, perhaps the most straightforward area of interaction to explore is between a person's physical and perceptual limitations and their response selection. For example, a number of digital human modeling approaches already include tools to identify constraints on performance such as range of motion, posture and strength capability assessment. When placed in a virtual environment, most digital human form models can be used to analyze reach and clearance envelopes as well as determine the field of view at a given moment. Some of these modeling approaches can generate realistic motion paths of the limbs and the whole body; many can also detect collisions between the person and an object. However, other than the modeling approaches mentioned at the outset of this section (e.g., HOS, MIDAS), the digital human models rarely, if ever, utilize any of this information internally to monitor physical safety and alter its performance in real-time. For example, what will most digital human models do if they encounter a load exceeding strength capabilities? Nothing different; the model will perform as scripted even if the task requirements exceed physical capabilities. Rather, safety and performance issues are identified mostly post-hoc.

Information Acquisition. On the other side of this interaction is the need of the cognitive system to acquire information in support of current activities. Most cognitive models assume that the information needed to complete the task being modeled is readily accessible, either directly or using some standard sequence of motor actions along with perfect knowledge of the location of the information source. But the role of search and eye movements in acquiring information is not a trivial issue. Add any need for physical actions other than eye movements to assist in the search process (e.g., postural or position changes, rearrangement of objects in the environment, etc.) and the impact is magnified further. Or, if information sources are present, but cannot be reached or inadequate clearance is present for a person to access them, the overall task of information acquisition will fail. In fact, Gray and Boehm-Davis [19] argue that a person is capable of selecting between different strategies for completing a task step on the basis of differences as small as 150 milliseconds. Further, they show that subtle differences in the location of information sources can bring about changes in which strategy is selected, resulting in significant effects on task performance. By utilizing the ability of the digital human model to track body position and posture relative to equipment in the environment as well as 
the body movements required to access information sources, one can account for the interaction between body position and information access. In many scenarios, this would reduce the variation between predicted and actual performance measures.

Errors. Another area of interaction to explore between digital human and cognitive models is the impact of errors. Given that most digital human models are scripted for performance, movements tend to be targeted and executed perfectly. Yet, as we all know, perfect performance rarely occurs in real life. And when the wrong button is pressed or an object is knocked off the desk, etc., we typically have to do something to recover from this error. This interaction between the imprecision of human movement and the need for cognitive strategies to recover from any resulting errors has a major impact on our ability to predict true human performance accurately. From the cognitive side, errors in navigation, decision making and information acquisition often add layers of complexity to the physical requirements of a task which, among other things, can lead to even more errors, higher likelihoods of failure, longer completion times, fatigue, and even injuries.

\subsubsection{Physiological Interactions}

Another major area of interactions is the effect of physiological influences on cognitive and physical performance. Human behavior is rife with apparently extraneous movements as itches are scratched, noses are blown, and postures are changed to improve comfort in response to physiological triggers. These unintentional behaviors produce variations in body positions and activity sequences that impact overall task performance. Increased heart rate and respiration act as indicators for localized and whole body fatigue; but whereas both physical and cognitive task performance are known to degrade with fatigue and can be accounted for in performance models, shouldn't models also account for any physical actions counter to the cognitive performance goals that become necessary to prevent physical harm and aid recovery? Temperature effects on both physical and cognitive performance are well-documented; but once again, physical actions counter to the cognitive performance goals may also be necessary. To complicate this even further, what strategies, if any, do people use for deciding when and what physiological actions to interject in a task?

\subsubsection{Emotional Interactions}

The most difficult interactions to consider will involve the influence of emotion on physical and cognitive performance. Anecdotally, strong negative emotions such as anger, fear and frustration often lead to actions that degrade task performance (e.g., performing too quickly, incorrect strategy selection, quitting tasks before completion or performing actions unrelated to any task at hand). Methods such as facial gesture recognition and physiological monitoring hold promise for identifying general emotions during human task performance (e.g., augmented cognition methods as described in Schmorrow [20]), but ethical issues involved in provoking these strong negative emotions in a controlled laboratory setting will need to be addressed before models accounting for the interaction of these emotions and task performance can be developed. 


\section{Conclusion}

Ultimately, we are looking for human models that, among other things, can adapt to the environment, get tired, make mistakes and become frustrated or angry - just like we do. Clearly, a lot of work is yet to be done. The empirical studies and modeling efforts required will be time-consuming enterprises and, as noted at the outset, are among the most difficult tasks a researcher could choose to undertake. But, modeling human performance - whether the physical form, the cognitive function, or some other aspect - is rapidly approaching a point where efforts should not (and perhaps cannot) be undertaken without collaborative efforts to put the results in the proper human context and to understand the inevitable interactions. Despite these challenges, this is a fascinating and gratifying field in which to work and, through all of these efforts - collaborative and otherwise - we should be able to watch our current human models grow from infancy and perhaps even reach adulthood.

\section{References}

1. Pew, R.W., Mavor, A.: Modeling human and organizational behavior: Applications to military simulations. National Academy Press, Washington DC (1998)

2. Baron, S., Kruser, D., Huey, B. (eds.): Quantitative modeling of human performance in complex, dynamic systems. National Academy Press, Washington, D.C (1990)

3. Elkind, J.I., Card, S.K., Hochberg, J., Huey, B.M. (eds.): Human performance models for computer-aided engineering. Academic Press, Inc., San Diego (1990)

4. Newell, A.: Unified theories of cognition. Harvard University Press, Cambridge, MA (1990)

5. Anderson, J.R., Lebiere, C. (eds.): Atomic components of thought. Lawrence Erlbaum, Hillsdale, NJ (1998)

6. Laughery, K., Corker, K.: Computer modeling and simulation. In: Salvendy, G. (ed.) Handbook of Human Factors and Ergonomics, 2nd edn., Wiley, New York (1997)

7. Kieras, D.E., Meyer, D.E.: An overview of the EPIC architecture for cognition and performance with application to human-computer interaction. Human-Computer Interaction 12(4), 391-438 (1997)

8. Liu, Y., Feyen, R., Tsimhoni, O.: Queuing network-model human processor (QN-MHP): A computational architecture for multi-task performance in human-machine systems. ACM Transactions on Computer-Human Interaction 13(1), 37-70 (2006)

9. Sheridan, T.: Humans and automation: System design and research issues. HFES Publications, Santa Monica, CA (2002)

10. Baron., et al. (1990)

11. Gluck, K.A., Pew, R.W.: Modeling human behavior with integrated architectures: Comparison, evaluation, and validation. Lawrence Erlbaum, Hillsdale, NJ (2005)

12. Lane, N.E., Strieb, M.I., Glenn, T.A., Wherry, P.A.: The human operator simulator: An overview. In: Moraal, J., Kraiss, K.F. (eds.) Manned System Design: Methods, Equipment, and Applications, Plenum Press, New York (1981)

13. Laughery and Corker (1997)

14. Bernard, M., Xavier, P., Wolfenbarger, P., Hart, D., Waymire, R., Glickman, M., Gardner, M.: Psychologically plausible cognitive models for simulating interactive human behaviors. In: Proceedings of the 49th Annual Meeting of the Human Factors and Ergonomics Society, 2005, pp. 1205-1210 (2005) 
15. Van Eerde, W., Thierry, H.: Vroom's expectancy models and work-related criteria: A meta-analysis. Journal of Applied Psychology 81(5), 575-586 (1996)

16. Vroom, V.: Work and motivation. Wiley, New York (1964)

17. Kernan, M.C., Lord, R.G.: Effects of valence, expectancies, and goal performance discrepancies in single and multiple goal environments. Journal of Applied Psychology 75(2), 194-203 (1990)

18. Klein, G.A.: Recognition-primed decisions. Advances in man-machine systems research 5, 47-92 (1989)

19. Gray, W.D., Boehm-Davis, D.A.: Milliseconds matter: an introduction to microstrategies and to their use in describing and predicting interactive behavior. Journal of Experiment Psychology: Applied 6(4), 322-335 (2000)

20. Schmorrow, D.: Foundations of augmented cognition. Lawrence Erlbaum, Hillsdale, NJ (2005) 